This item was submitted to Loughborough's Research Repository by the author.

Items in Figshare are protected by copyright, with all rights reserved, unless otherwise indicated.

\title{
Medical uncertainty and clinician-athlete relations: the management of concussion injuries in rugby union
}

PLEASE CITE THE PUBLISHED VERSION

http://journals.humankinetics.com/AcuCustom/Sitename/Documents/Documentltem/17191.pdf

\section{PUBLISHER}

(c) Human Kinetics, Inc.

\section{VERSION}

AM (Accepted Manuscript)

\section{LICENCE}

CC BY-NC-ND 4.0

\section{REPOSITORY RECORD}

Malcolm, Dominic. 2019. "Medical Uncertainty and Clinician-athlete Relations: The Management of Concussion Injuries in Rugby Union”. figshare. https://hdl.handle.net/2134/15315. 
This item was submitted to Loughborough's Institutional Repository (https://dspace.lboro.ac.uk/) by the author and is made available under the following Creative Commons Licence conditions.

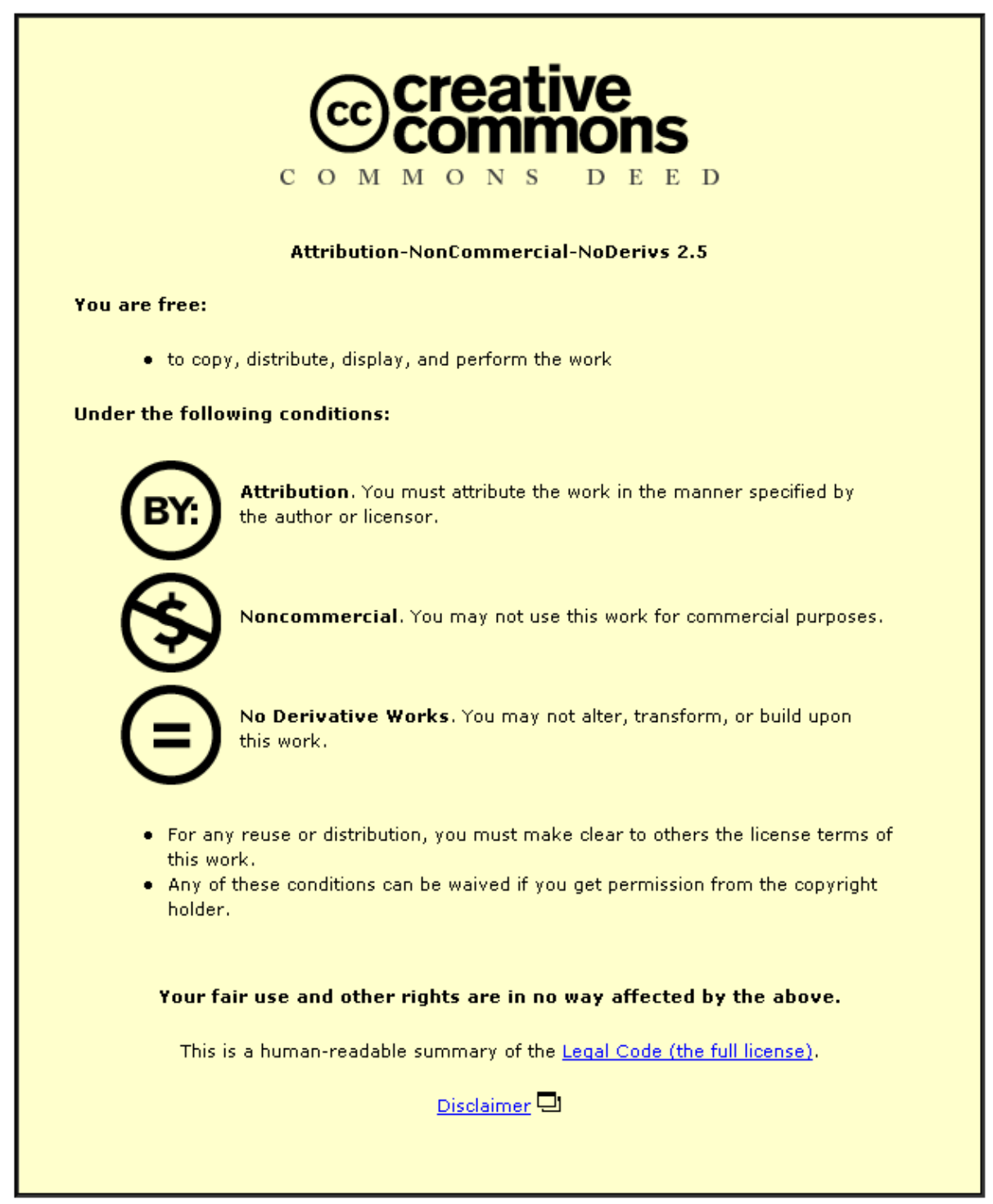

For the full text of this licence, please go to: http://creativecommons.org/licenses/by-nc-nd/2.5/ 


\section{Medical Uncertainty and Clinician-Athlete Relations: The Management of}

\section{Concussion Injuries in Rugby Union}

This paper addresses clinical practice in sport medicine. Combining notions of medical uncertainty with a figurational sociological emphasis on interdependence, the paper illustrates how uncertainty characterizes the medical understanding, clinical treatment, and patient experience of concussion. Faced with uncertainty, the clinician's desire for recognition and validation through athletes' dependence on them enables medically-based diagnostic and treatment guidelines to be replaced by the understanding and definition of concussion dominant in the sport subculture. Clinicians further invoke strategies that protect their professional status and therefore secure their interdependence with others in the sport club figuration. The paper advances our understanding by illuminating the basis on which clinicians and athletes negotiate treatment and the impact of these experiences on clinicians' actions and beliefs. 
The aim of this paper is to contribute to the emerging literature on clinical practice in sport medicine. Much of this work has concentrated on the "peculiarity" of sport as a context in which to practice (Walk, 1997). It focuses on the way that sport clinicians (a term used to encompass doctors, physiotherapists and student athletic trainers) are required to balance normal medical ethical considerations with the demands of employers and/or coaches (Waddington, 2000; Waddington \& Roderick, 2002), considerations of “precaution” and “risk” (Safai, 2003, 2004), and health relative to performance (Theberge, 2006). Malcolm (2006a) further illustrates why sport physicians often wield relatively limited power over their athlete-patients and physiotherapist colleagues, and Theberge (2008, p. 19) examines the "ongoing tensions" between different groups of health care providers within a "system of health professions.”

Walk (1997) and Safai (2004) indicate that coaches in North American college sport sometimes dispute or negotiate medical recommendations. Theberge’s (2006, 2008) research with clinicians working with Canadian Olympic athletes reveals a more harmonious but still discursive approach. Waddington’s (2000, pp. 71-79) work in relation to English League football suggests that challenges to clinical autonomy might be most prevalent in professional sport. The differences between the North American and UK literature do, however, indicate that the specifics of the "everyday work setting” significantly influence the treatment athletes receive (Malcolm, 2006a). The negotiation of health care in sport might be universal, but the relative power of negotiating parties varies according to the dynamics of the particular figuration (Elias, 1978).

The literature on clinical practice in sport medicine has grown out of the sociological work on pain and injury in sport (see Young, 2004 and Roderick, 2006a 
for overviews). Young classifies this literature in terms of Pain Cultures (the causes and contexts of injury), Pain Zones (athletes' reflections on injury rates and the experience of injury), and Pain Parameters (media, legal, and policy responses to injury). Summarizing the literature Young invokes ideas such as the "normalization" and "rationalization" of risk, pain and injury (Curry, 1993) as part of the "sport ethic" (Hughes \& Coakley, 1991), or “culture of risk” (Nixon, 1992). Gendered identities are also highlighted as significant in the social construction of injury (Messner, 1992; Young \& White, 2000), but regardless of gender, athletes tolerate injury "in order to maintain their athletic self” (Pike \& Maguire, 2003, p. 245). Young concludes that, “At the professional level at least, injury may thus be understood as the outcome of intricate relationships” (p. 6, emphasis added). Athletes talk about their bodies and understand injury in subculture-specific ways as a consequence of the networks of relationships that characterize the sport context.

This paper examines how networks of relations also influence the social construction of sport clinicians' knowledge. I argue that the social relations in which doctors are enmeshed influence not just what treatment they provide, but also the ways that they come to think about, understand, and define clinical conditions. The analysis combines the concept of uncertainty with a figurational sociological emphasis on interdependence. The paper contributes to our understanding of clinical practice in sport by highlighting the strategies practitioners use when uncertainty threatens their professional status and thus their patients' reliance on their expertise.

\section{Theoretical Perspective: Medical Uncertainty and Figurational Sociology}

Renee Fox (2000, p. 409), a leading sociologist of medicine, argues that “uncertainty is inherent in medicine.” Uncertainty has, moreover, become "a major 
motif in the medical sociological literature" because of the various ways in which it is manifest in medical practice.

Uncertainty is a common characteristic of being a patient and is therefore central to "illness experience” research (Conrad, 1987). Conrad suggests that patients may experience five forms of uncertainty. Patients experience uncertainty because: a) something unusual is happening to their bodies; and b) by definition, they have a problem that they themselves cannot resolve. Patients who believe that medical staff also harbor uncertainties about their condition will themselves become increasingly uncertain. Conrad places his final three categories under the umbrella term existential uncertainty. Existential uncertainty refers to "the individual's awareness that his or her future is open and undetermined” (Adamson, 1997, p. 134). This umbrella category can include trajectory uncertainty (where recovery is unpredictable), symptomatic uncertainty (where different symptoms occur at different times and in response to different stimuli) and, most centrally, uncertainty over how illness may effect one's life more broadly. The injured athlete might, for instance, worry about the possible end of a playing career and question self-identity (Roderick, 2004, 2006b).

Uncertainty is also a normal feature of becoming and being a medical practitioner. Fox (1957) argues that medical students experience three basic types of uncertainty: uncertainty stemming from the limitations of medical knowledge (epistemological uncertainty); uncertainty stemming from the awareness of being unable to master all aspects of medical knowledge and practice (clinical uncertainty); and uncertainty stemming from the inability to distinguish between the two. Aspirant medical practitioners therefore "train for uncertainty" through, for example, a process of intellectualization that entails the acquisition of greater knowledge and developing and applying methods for assessing probabilities to problems for which uncertainty 
exists (Fox, 2000, p. 410). These techniques provide some amelioration but "uncertainty is still attached to many aspects of medical practice” (Cockerham, 2004, p. 217$).^{1}$

A doctor's ability to manage their uncertainty is important for, as Adamson notes, "in many situations, expressions of uncertainty by medical professionals would violate norms and invite punitive sanctions” (1997, p. 135). Light (1979) argues that post-training physicians seek to increase control over clinical uncertainties through the assertion of individualized judgments made on the basis of personal experience, and by adopting particular treatment paradigms. Rafalovich (2005) identifies ways in which epistemological uncertainty can lead clinicians to be ambivalent towards diagnostic criteria. His study of the treatment of attention deficit hyperactivity disorder (ADHD) found that practitioners become more flexible in their implementation of diagnostic and treatment protocols when faced with patient/parent resistance, which has itself been fuelled by broader public debate over the condition. Clinicians' interpretations and applications of diagnostic labels may, consequently, "be a far cry from how texts provide the confines for a diagnosis" (p. 306). Yair (2007) argues that "under conditions of uncertainty, doctors chose to conform namely, to chose what others have elected - so as to gain social validation” (p. 689).

Use of a figurational sociological approach enables these largely microsociological processes to be linked to broader, macro-sociological factors. Elias's (1978) concept of "figuration" stems from a desire to overcome the traditional dichotomy between agency and structure. For Elias the "individual" and the "society" are two inseparable levels of the same human world and thus the key to understanding social phenomena is the examination of the "networks of interdependency" that humans form. In this analysis, therefore, I examine not just the face-to-face 
interactions experienced by sport clinicians, but consider the impact of a broader network of relations that encompasses rugby administrators and the community of sport medicine researchers. Elias, moreover, views power as a structural property of all social relations, always a question of balance and always in flux. In this paper, therefore, I examine the process by which sport medicine personnel are enabled and constrained to act and react to others in order to safeguard their status and position within the "rugby club" figuration. The concept of medical uncertainty, moreover, resonates with Elias's understanding of figurations as highly complex and thus of human understanding of the social world as consequently and necessarily incomplete. Figurational sociologists have traditionally identified the co-existence of intended and unintended consequences as the main manifestation of this (e.g. Murphy \& Sheard, 2008), but my focus here is on the way that the doubt generated in complex networks of social relations leads to "internal” self-regulation and behavioral adaptation.

Following a discussion of research methods, I examine how epistemological, clinical, and existential uncertainty influence the behavior of injured players and sport clinicians. These three domains of medical uncertainty are presented separately in the first part of this paper, though in keeping with the figurational approach, they should be seen as highly interdependent. The paper subsequently draws on the literature on the management of medical uncertainty to illustrate how sport clinicians manage concussion injuries in rugby so as to minimize conflict with their athlete-patients. Clinicians adapt their practice to foster cooperation and thereby enable their interdependent relationships with coaches and players to continue. 


\section{Methods}

Data were collected as part of a broader project on the management of injuries in English elite male rugby union. "Elite" rugby union was defined as the 68 teams playing in the top four Leagues. ${ }^{2}$ I have explored the effects of commercialization on the treatment of sporting injury (Malcolm \& Sheard, 2002), critiqued epidemiological work which falsely portrayed an "injury crisis" in rugby union (Malcolm, Sheard \& Smith, 2004, 2005), examined the changing character of sports medicine provision in the game (Malcolm 2006b), and sought to explain why sport clinicians wield relatively little influence in professional sport (Malcolm 2006a). The research included both a questionnaire survey and semi-structured interviews, though it is the latter that centrally informs the analysis here.

Between September 2000 and October 2001, a colleague and I interviewed nine doctors, ten physiotherapists, sixteen players, and seven coaches. The sample was assembled using largely convenience and snowball sampling methods and included representatives from fifteen of the 68 clubs. Some purposive sampling was used to ensure the sample included respondents from a geographically dispersed range of clubs and clubs with less complex organizational structures. Non-probability sampling methods were justified on the basis that no reliable sampling frame was available (de Vaus, 1996, p. 77), and because those who work in professional sport are notoriously “elusive” populations to access (May, 2001, p. 95).

Interviews lasted between 30 and 80 minutes and took place in various locations including workplaces, homes and rugby clubs. Interviews were recorded and transcribed in full. Interview schedules covering key areas for each category of interviewee were drawn up using the existing literature (especially the work of Roderick, Waddington \& Parker, 2000), and the researchers' existing knowledge of 
the game. Doctors and physiotherapists, for instance, were asked about how they obtained their position at the club, their normal work routine, and how commercialization processes were influencing their clinical practice (see Malcolm \& Sheard, 2002). They were further asked about their relations with players, coaches and other medical staff. The emphasis on interdependence, and the way that structural changes in the sport impacted upon aspects of agency, developed out of a commitment to a figurational sociological approach.

Three topics of potential conflict in these relationships were identified: painkilling injections, confidentiality and concussion. It became apparent during the research process that clinicians saw dealing with concussion as the most problematic aspect of their practice. The flexibility of the semi-structured approach gave interviewees considerable scope to develop this topic. Data on concussion were abstracted from the interview transcripts and themes identified according to their frequency (Stroh, 2000, pp. 210-212). An additional colleague suggested that research on medical uncertainty might be helpful in developing the analysis of concussion management, and this led to a process of cross-referencing of empirical data with the literature from sport medicine and the sociology of medicine.

In the next sections I outline how uncertainty permeates the medical understanding, clinical treatment, and patient experience of concussion. The debates about concussion in medical journals illustrate the limits of medical knowledge and therefore epistemological uncertainty. Regulations in rugby union, moreover, are explicitly justified on the basis that head injuries often entail considerable clinical uncertainty. I then use testimony from doctors and physiotherapists to show not only that many experience clinical uncertainty when dealing with concussion, but attempt to intellectualize and/or rationalize the condition and their treatment of it, before 
describing how concussion entails specific forms of existential uncertainty for the injured player. The paper concludes by illustrating how clinicians, when faced with these co-existing forms of uncertainty, reject medical guidelines and pursue strategies that will minimize conflict and thus secure their position in the "rugby club" figuration.

\section{Epistemological Uncertainty and Concussion}

Concussion is typically caused by rapid acceleration, deceleration, or rotation of the head. It normally entails the sudden onset of symptoms at the point of impact, that are often short-lived and resolved spontaneously (McCrory, Johnston, Meeuwisse, Aubry, Cantu, Dvorak, Graf-Baumann, Kelly, Lovell \& Schamasch, 2005, p. 196). There are three main symptoms of concussion - loss of consciousness, loss of memory, and confusion - with loss of consciousness traditionally seen as the primary measure of severity. The International Rugby Board (IRB) also identifies giddiness, unsteadiness, vomiting, disorientation, headache, and double or blurred vision as “signs and symptoms of concussion.” Regulation 10.1.1 concludes that, "being unaware of what happened, even for a few moments at the time of the injury is the most consistent sign that the player is or has been concussed” (IRB, 2008).

The IRB has adopted a definition of concussion that does not differentiate between grades of severity. Any player sustaining any concussion must abstain from playing and training "for a minimum period of three weeks," and should only resume "when symptom free and declared fit after a proper medical examination.” This period may only be foreshortened following examination by a recognized neurological expert. $^{3}$ The rationale for this relatively strict policy is identified in the (English) Rugby Football Union's (RFU) Handbook of Safe Rugby (RFU, 1998) which states 
that the IRB ruling is based on "the need to have a simple and universally accepted instruction across the whole spectrum of world rugby, which is applicable by those without medical training” (p. 51).

Concussion is the only injury that the IRB (2008) seeks to define and, with blood injuries, the only injury subject to specific regulation. Concussion, moreover, is one of the IRB's seven "first priority" areas for medical research funding. These policy stances are indicative of the concern over the harm that can potentially be caused by such injuries. The "real danger" of concussion, the RFU (1998) state, is often obscured and seemingly mild concussions "may rapidly progress to become life threatening” (p. 47). Paul McCrory (2001a), a leading sport neurologist, notes that the heightened concern over concussion stems from the notion that repeated incidents predispose athletes to "second impact syndrome;" where subsequent head injuries become increasingly regular and/or severe (p. 381). McCrory also refers to the existence of an "unstated fear" that athletes who are repeatedly concussed will ultimately experience similar cognitive decline to that of the "punch drunk" boxer (p. 380). Whilst the IRB has particularly strict policies, debates about concussion in other sports indicate a more general perception of the severity of this kind of injury (see, for example, Schwarz, 2007).

The sport medicine community has begun to voice significant epistemological uncertainty regarding sport-related concussion in recent years. This gap in knowledge stems from the absence of data relating to contact sports for, "there is no existing animal or other experimental model that accurately reflects a sporting concussive injury” (McCrory et al., 2005, p. 197). Existing studies commonly draw upon the literature on head injuries sustained in boxing and motor vehicle accidents. These comparisons are, however, misleading because: a) the frequency of repetitive head 
trauma in boxing is thought to pose "unique risks;" and b) collisions in sports such as rugby union involve much lower acceleration-deceleration forces than do motor vehicle accidents. There has been some debate over whether to grade concussion injuries according to duration of symptoms rather than on the presence/absence of particular symptoms (McCrory et al., 2005). There is, moreover, no scientific evidence that sustaining several concussions over a sporting career will necessarily result in permanent damage (McCrory, 2001a). McCrory (1999) argues that the proliferation of scales designed to assess the severity of head injuries means that clinicians and athletes are often confused by conflicting advice and thus illustrates how epistemological and clinical uncertainty interact.

McCrory (2001b) criticizes existing research on concussion for being “anecdotal ... bizarre rather than reflecting established medical principles” (p. 82). He concludes that the field is plagued by "neuromythology" derived from folk wisdom, methodologically flawed medical research, and media stories of athletes' experiences of head trauma. Even the 2004 Prague Agreement Statement on Concussion in Sport, authored by ten of the world's leading experts, concedes that "the science of concussion is at an early stage” (McCrory et al., 2005, p. 202). This acknowledgement has also had the unintended consequence of conveying epistemological uncertainty to the practice domain. It is to an illustration of clinicians' uncertainty over concussion that I now turn.

\section{Clinical Uncertainty and Concussion}

Research in the sociology of medicine indicates that doctors are often reluctant to express uncertainty for fear of undermining their expert authority (Calnan, 1984; Adamson, 1997; Rafalovich, 2005). Physiotherapists, given their respective 
professional status, might be more inclined to disclose their concerns. However the majority of sport medicine personnel interviewed, regardless of their profession, expressed elements of uncertainty about their own knowledge of concussion. Their strategies for overcoming uncertainty not only illustrate what a problem this is for practitioners, but also their interdependence within a broader network of relations of sport administrators and the sport medicine community.

Clinicians normally responded to questions about concussion by describing the condition as "Very, very, difficult” (physiotherapist), “a very dodgy area” (doctor), or a "huge grey area" (doctor). One doctor indicated that concussion had been discussed with fellow clinicians: "What's concussion is an issue that's come up." The doctor went on to note that, "I know it's not pain," but did not provide a more definitive answer. A physiotherapist not only noted the problems they had with the diagnosis of concussion but argued that, "In fact the doctors are confused too."

Interviewees sometimes rationalized their own uncertainty by responding to questions about concussion with questions of their own. One doctor, who argued that loss of consciousness provided a relatively reliably diagnostic guide, continued, "But when a guy is banged on the head and is a bit dizzy and feels sick for a few hours, is that concussion?” Another doctor, reflecting on a particular incident, said, "I suppose on a very low scale you could diagnose, there was probably a bit of concussion, but what is concussion?” The degree of uncertainty was most clearly demonstrated by a physiotherapist who was asked to clarify their continued use of the term "head injury.” The physiotherapist replied, “we've had some players who have not necessarily, obviously, had a concussion, but have had some form of concussion symptoms, so I use ... (long pause) well they don't have concussion but they have 
symptoms of a head injury.” Following a further pause, the physiotherapist asked, "Should I be defining that as concussion?"

Clinicians sought to legitimize their uncertainty by referring to the conditions under which they were required to practice. One doctor argued that, "the clinical fact is all laid out - how you go about diagnosis - the only problem is the player's taken a (mild) knock and in the heat of the situation it's really quite pressured.” Another stated that the "problem is that you have to make a snap decision," and further called for regulations to provide more time to assess head injuries. A third doctor argued that, "it is virtually impossible to check the extent of a head injury in a dehydrated player.” A fourth identified the cognitive testing done in Australian Rules Football as a potential advance and explicitly argued that the central benefit of this would be to alleviate uncertainty: "then you can be certain that they returned to pre-injury brain function.”

Club doctors, like the junior doctors studied by Fox (1957), typically attempted to overcome clinical uncertainty through a process of investigation and intellectualization. One doctor argued that, "I looked into this when we had a guy with a bang on the head ... There is no definition that is widely accepted; there are a variety of definitions all of which are slightly different.” Other doctors had sought direction from the national governing body, the RFU, but remained dissatisfied: "we did get them on the phone but when I'd finished talking to them I thought now was that very helpful at all or what?” Another stated that,

I spoke to the RFU doctor on this and he agreed with me that it's all a bit up in the air. Nobody seems to be interested in changing it or making it more scientific. We spent a lot of time saying it isn’t scientific and how there isn't a definition but nobody seems to be trying to get anything more sensitive. 
The attempts of doctors to alleviate their clinical uncertainty were, however, undermined by the epistemological uncertainty evident in the broader sport medicine community. One doctor noted that, "The number of conferences about concussion you wouldn't believe," and went on to argue that "there are suggestions that there's no common factor that helps you say that was most definitely concussed, or certainly not concussed.” Another pointed to the irrelevance of existing severity scales to sport: 'All the scores ... are designed for serious head injuries, not just a bit of mild concussion and usually the relevance to somebody feeling a bit dizzy after a game is zero.” The doctor concluded that, "we have no way of assessing the severity (of a head injury) in that minor bracket.” The network of relations that clinicians normally find enabling - that is to say, their association with the researchers who provide the knowledge base of their profession - are thus unreliable in the case of concussion.

The disclosure by so many clinicians participating in this study that they have been unable to master this area of medical practice indicates how widespread clinical uncertainty over concussion is. Concussion also throws into sharper relief the dependence of club doctors on the broader network of relations. Clinicians will seek to resolve uncertainty through discussions with each other, sport administrators and, perhaps most importantly, consultation with the community of sport medicine researchers. Clinical uncertainty and epistemological uncertainty therefore come to intertwine. An examination of the dynamics of the treatment of concussion, however, also requires an understanding of the existential uncertainties that players with concussion injuries experience for "clinical and existential reactions to uncertainty play to and play off each other in all sorts of ways” (Adamson, 1997, p. 154). It is to players’ existential uncertainty that I now turn. 


\section{Existential Uncertainty and Concussion}

Aspects of existential uncertainty pre-occupy the thoughts of the injured athlete (Roderick, 2004). The injured may be stigmatized by their peers and "non-producing” athletes labeled as malingerers. Professional athletes' dependence on coaches means that they live with the constant fear that injury will lead to demotion, enforced relocation, or career termination. Roderick (2006b) argues that professional soccer players use three coping mechanisms to deal with injury-induced uncertainty: a) seeking second opinions; b) negotiating the timing of surgery or other remedial action; and c) constructing "treatment timetables" (p. 66). The first and second rely on the identification of compliant clinicians, whilst the third entails a process of athlete-clinician negotiation. Each coping mechanism gives the athlete-patient the feeling of progress and influence over their future recovery, thus allaying some of the uncertainties associated with injury. The existential uncertainty that arises from a concussion injury, in contrast, leads players to be relatively independent of, rather than more dependent on, clinicians. This, in turn, has ramifications for the actions, and ultimately the beliefs, that clinicians hold.

Existential uncertainty influences the ways that athletes define pain and injury. Whilst Young (2004) argues that there has been a lack of rigor in the attempts of sociologists of sport to differentiate between these concepts, Malcolm \& Sheard (2002) and Howe (2004) provide descriptions of how athletes, and rugby players in particular, distinguish these phenomena. Pain is often the "marker of injury" (Howe, p. 74) but pain can be experienced in the absence of injury, and one can be injured without necessarily being in pain; concussion being a case in point. Rugby players, Malcolm \& Sheard (p. 160) argue, see pain as a normal and inherent feature of the sport but, crucially, a condition that does not significantly impair functionality or 
sporting performance. Rugby players define injuries as physical conditions that curb their ability to perform to the extent that they are required to miss training and/or matches, as this is the point at which existential uncertainty becomes most pronounced.

Concussion injuries are unusual for, in contrast to soft tissue injuries that may gradually worsen over time, they occur instantaneously and thus a player's sense of existential uncertainty comes, literally, with a bang. The prescribed cessation of playing and training will, furthermore, often be at odds with a player's understanding of their ability to perform and thus "being injured.” Players and coaches do not see concussion as necessarily impairing functionality and some specifically noted that, despite being concussed, they had remained effective on the pitch. A coach and former player recalled "coming round" in the shower after a game but argued that, "I played a great game. I had a fantastic game.” A current player similarly argued that when concussed, “you don't feel as though there is anything wrong with you.” Illustrating the distinction that rugby players draw between pain and injury he went on to say, "I mean a week, a day, later (you) might have a sore head, but that's about it" (i.e. in pain but not, according to the player's definition, injured). Many seemed content to play in the weeks following concussion, a few played after sustaining concussions in consecutive matches. Some recognized that concussions posed a potential danger to longer-term health, but this risk seemed to be beyond the temporal horizons of most players. Concussed players therefore experience uncertainty because their bodies feel unusual, but they seldom experience problems that they themselves cannot resolve and rarely, or only briefly, experience uncertainty in the form of concern about sporting performance. 
A concussed player's existential uncertainty is, moreover, unlikely to be relieved through consultation with a clinician. Pharmacological treatment of concussion is only recommended in cases of prolonged symptoms (McCrory et al., 2005) and thus medical staff can offer little by way of remedial action. Clinicians will, more typically, merely consult with players to ensure that the condition is not worsening. One player illustrated this when referring to a game in which he had been noticeably disoriented: “the doctor came and said, 'You alright?’ and I said, 'I just feel a bit sore at the back of my head.' And he said 'Take it easy.'” Players cannot seek a second opinion because medical knowledge is not sufficiently developed for any person to retrospectively deny that a concussion injury occurred, and the neurological expertise required to clear someone to resume playing is too specialized to be widely accessible.

A concussed player's existential uncertainty is, however, likely to increase as a consequence of a medical consultation because clinicians are required to impose a three-week abstention from the game when they diagnose concussion. This might not entail trajectory uncertainty (quite the opposite, the recovery path is rarely so certain), but will produce uncertainties over team selection and, ultimately, what the injury “means” for a player's career. Concussion guidelines make irrelevant the treatment timetables that players normally invoke to deal with the uncertainty of being injured. A medical consultation for concussion will, therefore, contrast sharply with the "ideal medical encounter ... in which the physician is certain of the correctness of his or her diagnosis, and the patient obtains a clinical response which alleviates his or her feelings of existential uncertainty” (Adamson, 1997, p. 136).

Players are consequently reluctant to seek medical advice for head injuries that they can hide. A former player recalled that, "there have been times where both 
players and management have said, 'avoid the possibility of seeing a doctor and being diagnosed.' It's just a matter of keeping away.” A doctor noted that players often talked about "bell ringers,” or "amusing” incidents of concussion on the team bus. The doctor inferred from this that players rarely reported concussions to medical staff. A physiotherapist similarly noted:

One of my biggest problems at the club is that you lose head injuries. If someone has a head injury one weekend, you give them advice ... (but they) don’t come in to see you ... they just walk on to the training ground and they feel a bit better, so they just go and train.

Athlete reluctance to consult with medical staff is not unusual in sport, but concussion injuries in rugby union lead to rather peculiar forms of existential uncertainty and thus to a specific manifestation of this phenomenon. Players who receive concussions may find it difficult to justify an abstention from training to their self, and may consequently question, disregard or simply avoid being given medical advice. Medical staff can offer little in the way of treatment that can allay existential uncertainty and the regulatory framework leads medical staff, and their formal diagnosis of concussion, to significantly increase players' existential uncertainty. The uncertainty that concussed rugby players experience ultimately reduces their dependence on medical staff and thus clinicians find them particularly difficult to manage. Clinicians' actions are shaped in response to these patient-management problems. Clinicians implement diagnostic and treatment guidelines in ways that serve to counteract, or limit, the effects of this non-compliance. 


\section{The Clinical Operationalization of Concussion}

Doctors argued that concussions, and head injuries more generally, were a significant source of tension between themselves and players and coaches. One doctor argued that head injuries were "one of the things that I absolutely hate about the medical side of rugby at the moment.” A second more specifically noted that,

Quite often the player, within an hour, is back to normal, and with no neurological signs or symptoms, and the coach is wanting him to play next week. And it's head injuries, particularly, (that) can be a cause of conflict.

A third doctor recalled how a player who was withdrawn from a game exhibiting concussion symptoms refused to speak to them "for years," whilst a fourth stated that the only major clash that they had experienced stemmed from a player who was "obviously concussed." The doctor recalled that, "I told the coach that he had to come off and he initially said, 'I'll make that decision.'” Concussion may lead to some of the most pronounced conflicts over medical management because the clinician-patient relationship is likely to create more rather than less existential uncertainty for the player, and because the regulatory framework leads the diagnosis of concussion to have specific difficulties for coaches over selection. In both cases the involvement of a clinician is seen to hinder rather than help.

Clinicians' attempts to allay their own clinical uncertainty through a deeper investigation of concussion are, as highlighted above, largely unproductive because research only serves to throw into sharper relief epistemological uncertainty over the condition. This, combined with the resistance of players and coaches, leads to a rejection of treatment protocols. Clinicians come to diagnose concussion in a way that they come to know will be acceptable to others in this clinical setting. Because such a “compromise" is potentially threatening to their authoritative status, doctors in 
particular invoke strategies that serve to obscure their clinical uncertainty and thus reassert their expertise.

\section{Diagnosing Concussion in the Clinical Context}

Clinicians are enabled in adopting their own diagnostic paradigm (Light, 1979) as a consequence of the questioning of the medical evidence for IRB concussion guidelines in the sport medicine community. A physiotherapist stated that "the rule of thumb that says that you have to be out for three weeks is a bone of contention.” A doctor similarly noted that, "evidence for the three week ban is scanty to say the least.” Others pointed to the existence of regulatory differences between sports as evidence of the arbitrary nature of IRB policy. One doctor argued, "it's all a bit Mickey Mouse it seems to me. One sport saying, 'Oh yes, three weeks is alright' as if it's based on some sort of great scientific foundation when it patently isn't.” One might expect the rejection of a standard treatment philosophy to lead to considerable diagnostic variation, but the influence of subcultural norms leads to a distinct pattern in the way rugby clinicians diagnose concussion.

At the most "cautious” end of the spectrum concussion comes to be defined by the symptom that the medical literature has traditionally depicted as the most severe; that is to say, loss of consciousness. Doctors typically argued that, “if they haven't been knocked unconscious, they have not suffered concussion.” Some argued that there were “grey areas ... where they’ve taken a bit of a knock but they haven’t lost consciousness”. The diagnostic examples doctors gave, however, indicated that when called upon to make a clinical judgment, symptoms within the so-called "grey areas" did not lead to a diagnosis of concussion. A doctor spoke about a player who "was a 
bit woozy and a bit woolly headed for ten minutes but made a full recovery” and therefore wasn't diagnosed with concussion. Another gave the example of,

a heavy bang, a heavy collision ... (It may) sort of daze someone, and they may recover after three or four minutes, or their game may be effected for the next 40 or 50 minutes; they're not truly concussed, they're just sort of bashed.

Some even argued that a loss of consciousness was not, on its own, sufficient to diagnose concussion. In the view of one doctor, "if someone is knocked out and there were signs of neurological disturbance, then I would probably seriously think about diagnosing concussion” (emphasis added). Clinicians' rejection of the IRB guidelines and their underlying precautionary philosophy is enabled by their knowledge of the epistemological uncertainty about concussion.

Players' definitions of concussion are shaped by their experiences of existential uncertainty and thus also focus on loss of consciousness. One player noted that, “(I’ve) never had concussion. I've banged my head a few times, but I've never knocked myself out.” Another contrasted an instance that he defined as concussion "Out. Out completely" - with one that, despite the presence of symptoms such as confusion, blurred vision and disorientation, he did not consider concussion. The low number of interviewees who had been "officially” diagnosed as concussed illustrated that loss of consciousness was seen as a necessary rather than a sufficient diagnostic criterion. Though many argued that losing consciousness was something that most rugby players would have experienced a couple of times, just four of the sixteen players interviewed stated that they had undergone the proscribed three week cessation of playing and training. Three of these players had experienced a loss of consciousness, whilst the fourth player had a suspected fractured skull. In each case, 
therefore, an impairment to performance was evident and an increased dependence upon clinicians was unavoidable.

The perceptions that players, coaches and medical staff have of concussion therefore converge. Doctors made reference to the influence of subcultural norms in this regard. One doctor, though personally espousing a definition of concussion that encompassed other symptoms, noted that, "most people would define concussion, most rugby people, if a player was knocked out, even for a few seconds” (emphasis added). The doctor added "rugby players will say 'That's not concussed. He wasn’t knocked out."” Clinicians also explicitly recognized that their diagnosis was at times more influenced by the broader networks of relations than by their clinical understanding of the condition. One doctor, for instance, described an incident in which a player received a "significant" blow to the head. The following quotation illustrates how the doctor considered the potential consequences of a diagnosis in terms of the coach's team selection and the player's subsequent reaction to any existential uncertainty this might entail:

He lost his $1^{\text {st }}$ team place, the $2^{\text {nd }}$ team hooker took his place, played well and kept his place. And really he didn’t get his place back for the rest of the season. And that caused him to say to the head coach, "I don't want to stay in (club) I'm not getting $1^{\text {st }}$ team rugby." He's now left the club ... so you know a single injury can have a profound effect on a player's career. So you've got that to bear in mind, but as a Doctor you want to do what's right medically for the patient.

Since rugby club doctors practice in similar social contexts, or rather are enmeshed in similar networks of social relations or figurations, their diagnoses converge. Despite being at odds with IRB regulations and the sport medicine 
literature, the diagnosis of concussion comes to focus upon loss of consciousness. There are two main reasons for this. First, loss of consciousness is the most visible symptom of concussion. It is therefore the symptom about which the least uncertainty exists and the symptom that is most likely to increase a player's dependence upon clinicians. Second, loss of consciousness is the symptom traditionally seen as the most severe. The diagnosis of concussion entails a heightened state of existential uncertainty for a player and thus loss of consciousness is often the minimum symptom that rugby players are prepared to accept as indicative of a medical problem. Club doctors' uniformity of approach illustrates that the greater the degree of clinical uncertainty the greater the scope for broader social pressures to exert an influence over a clinical diagnosis. Clinicians ultimately adopt the definition of concussion most widely held by players and coaches because it enables medical consultations to continue. Clinicians prefer "to be existentially secure in a supporting social group rather than being empirically correct in isolation” (Yair, 2007, p. 687).

\section{Managing Concussion, Asserting Expertise and Professional Success}

Clinicians minimize interpersonal conflicts that might undermine their professional status by allowing the pressures stemming from the broader network of social relations to direct their diagnosis. The respect that clinicians might otherwise expect is, however, likely to be undermined if others do not perceive them to possess skills and techniques that are the preserve of their profession (Malcolm, 2006a). If clinicians are not respected, their patients are unlikely to feel dependent upon their expertise. Clinicians thus use three interconnected strategies to obscure their clinical uncertainty, (re-)establish expertise, and thus contribute to professional success by ensuring their clients' continued reliance upon them. These are: avoidance of 
diagnosis; the adoption of a personal treatment philosophy; and the individualization of cases.

One way that doctors can mask their clinical uncertainty is to avoid making a diagnosis. A central aspect of this strategy is to adopt the very narrow definition of concussion outlined above. In addition to using limited criteria, clinicians become reluctant to use the specific labeling. One doctor, for instance, argued that, "It's best not to diagnose it. It's best to have an opinion as to whether the player should be playing or not.” The physiotherapist at the same club similarly argued that, "you take them off if you suspect it (concussion), but we don't use the c-word unless we have to." A second doctor referred to "public concussions and keep-quiet concussions" whilst another spoke about a famous televised incident in which a player was "clearly concussed” but received no treatment: “The doctor's excuse was that he wasn't allowed on the pitch to make the diagnosis. He was hiding without (a) doubt.” Others provided personal accounts where a mixture of clinical uncertainty and competitive pressure combined to produce a non-diagnosis. Describing the diagnosis of concussion as “all a bit arbitrary,” a doctor was asked if a diagnosis depended on when the next important game was to be played:

Exactly! We had a guy, before the play-offs, and he had a bang on the head and was dizzy, and he was feeling a bit sick, but he didn't have amnesia and he didn’t have any neurological symptoms and I looked at it and said, “Alright, I don't have to diagnose this as concussion” ... and we let him play two weeks later.

Players confirmed the tendency of clinicians to avoid diagnosis. One player indicated that, when a concussion occurred at his club, the "player goes off but then it’s very quickly pointed out, 'no he’s not got concussion,' because as soon as you say 
he's got concussion then he's got to have three weeks off.” The player suggested that other considerations influenced the decision: “(this happens) particularly if they're a key player, but if he’s not a key player then he might play the next week and then he might wear a scrum cap.”

Clinicians were, however, required to provide a rationale for their actions when circumstances forced a diagnosis. In the absence of a literature that can alleviate clinical uncertainty, many of the doctors interviewed used personal experience to develop their own "guidelines" for diagnosing concussion. One identified a reliance on experiential knowledge: “I know the players well, I talk to them quietly, I'm watching their balance.” A second explicitly contrasted their actions with official guidelines: “There is obviously the clinical diagnosis but concussion, as far as I'm concerned, is the inability to think straight after a heavy collision; the brain is shutting down.” A third supplanted medical guidelines with an assessment of player welfare:

I want to make sure the player is safe enough to play rugby ... Is this guy able to take another hit? ... Is this guy safe to carry on playing rugby? And that's what I'm interested in, not a label.

Such rationales enable some diagnostic consistency. They also have the advantage that issues of clinical uncertainty are superseded by seemingly objective measurements of functionality. Allowing sporting performance criteria to override medical guidelines further enables the diagnosis to become consistent with rugby players' (and coaches') own definitions of what constitutes an injury.

An extension of developing personal treatment philosophies is the process of individualizing concussions. Doctors compared concussions to muscle tears, arguing that they "are not (all) the same; you can get a mild one and a more severe one." Some further argued that one had to take a player's recent history of concussion into 
account when making a decision. Clinicians also considered the way that players had responded to previous concussions as part of the process of the individualization of injury. One doctor argued that, "Each player responds differently. There are some who get knocked every week and are fine after a couple of minutes,” whilst the physiotherapist at the club described how individual case management had become semi-formalized:

We (the coaches and medical team) tend to have in our own minds ... a rough idea of the players who can take a knock on the head, be dazed, clearly concussed and recover quite quickly and can then go on and play and don't seem to suffer any long-term effects. We also have a number of other players who tend to suffer far more profoundly from blows to the head and they're the ones we tend to monitor far more carefully.

These responses to, and understandings of, concussion are far removed from the sport's regulations. The protectionist philosophy underpinning IRB guidelines has the unintended consequence of leading clinicians to avoid the diagnosis of concussion. The rationalization of personal treatment philosophies and individual case management provide the appearance of considered medical assessment and objectivity and thus hide the socially constructed character of clinicians' understandings of concussion. The strategy of individualizing cases and allowing sporting performance criteria to dictate "fitness to play" decisions, effectively minimizes the potential for interpersonal conflict and thus preserves the clinicians' professional status by facilitating the continuation of a collaborative relationship with the patient-player. 


\section{Discussion and Conclusion}

In this article I have drawn upon the understanding of uncertainty within the sociology of medicine and applied it to the medical understanding, clinical treatment and patient experience of concussion in rugby union. I argue that not only clinicians' behavior, but also their understanding of medical conditions, is shaped by the interdependent relationships in which they are enmeshed. Clinicians cannot access support from their broader professional network in the form of reliable medical knowledge and, because conflict with playing and coaching staff might undermine their position, come to internalize a definition of concussion that is similar to the way players and coaches understand the condition. This is not simply a conscious avoidance of a problematic medical issue, for the process entails an element of internal self-adaptation and the adoption of beliefs generated in a way that is starkly at odds with the conventions and formal basis of medical practice. In order to ensure that this “concession” does not threaten their professional status, clinicians invoke a number of strategies that serve to mask their clinical uncertainty and provide the appearance of considered medical assessment. Clinicians therefore avoid being marginalized and remain part of the network of interdependent relationships that constitute the sport club.

This research develops concepts such as the "normalization" and "rationalization" of pain and injury in sport by more explicitly connecting them with existential uncertainty. Injured athletes may rationalize their continued participation in sport or normalize physical ailments not simply because the sport ethic or culture of risk “demands" that they do so, but because these actions partly allay feelings of existential uncertainty. This paper also builds on Roderick’s (2004, 2006b) discussion of existential uncertainty by identifying that emotional state as contingent not only 
upon the relationships athletes form with coaches, but also with clinicians. Existential uncertainty is contoured by the interdependent relationships in which any individual is enmeshed.

This analysis, moreover, enhances our understanding of the clinician-athlete negotiation process described in much of the literature on clinical practice in sport medicine. The literature has largely portrayed clinicians as confident in their own knowledge yet frustrated by their inability to gain compliance from their athletepatients. This study of concussion, in contrast, highlights the insecurities clinicians experience and thus the sometimes weak evidential basis of their negotiating position. Clinicians' willingness and ability to adopt beliefs about a medical condition that are partly at odds with the sport medicine literature and wholly against the spirit of IRB regulations, shows how fundamentally effected they are by their involvement in these networks of relations. Where athletes tolerate injuries in order to maintain their athletic self (Pike \& Maguire, 2003), clinicians tolerate “compromised” diagnoses to maintain their medical self.

The use of the concept of uncertainty, and the figurational sociological emphasis on interdependence, may also therefore broaden understanding of the treatment of injuries in sport. Concussion is clearly rather different to the soft tissue injuries that athletes most commonly experience. Its potential severity is offset by its short-lived nature and (often) spontaneous resolution and concussion policies in rugby union, but also across sport, are relatively proscriptive. Players and clinicians may therefore experience different degrees of uncertainty to that experienced in relation to other types of injury. It is not the case however that this uncertainty is different in kind. As Kevin Young (1993) has argued, "sports workplaces are simultaneously sites of medical mastery and extraordinary medical neglect” (p. 376). Uncertainty is likely 
to be a regular, and heightened, experience for clinicians at this cutting edge of medicine, where innovation and experimentation are driven by the search for performance-enhancement (Theberge, 2006). Clinicians can never be sure whether, for instance, playing with a damaged muscle will lead to further harm, and negotiations over treatment will centrally involve the interplay of this clinical uncertainty and the athlete's existential uncertainty. Clinicians' management of uncertainty will profoundly influence the degree to which athletes perceive medical consultations to be beneficial. This, in turn, will influence the degree to which clinicians can persuade athletes to comply with their advice.

The illustration in this study that clinicians may sometimes prioritize selfrespect within the rugby club over medical considerations highlights the importance of interdependence to the understanding of human relations. Where clinicians are less isolated and work more closely with other medically qualified personnel (Malcolm, 2006b), they may experience interdependencies that counteract the strong dependence upon the evaluation of their "clients” evident here (Malcolm, 2006a). Clinicians' actions will be less influenced by broader social pressures when they have confidence that the research-orientated members of their profession have produced relatively reliable knowledge that supports their actions. Elias's conception of interdependence is of value, thus, because it enables the analysis to move beyond the face-to-face clinician-athlete interactions, and to encompass the broader constituencies from which people draw occupational status and emotional support, and thus draw aspects of their identity.

I do not claim that this paper expands Elias's theoretical model. The focus on clinician-athlete relations, and in particular the exercise and contestation of knowledge within these relations, is compatible with Elias's emphasis on the 
polymorphous nature of power, and the specificity of power relations to different contexts. Figurational sociologists of sport have not previously used the concept of uncertainty to illustrate the limited human understanding of the social world that necessarily stems from the complexity of figurations, but again I see this as an expansion rather than a revision of Elias's existing theoretical framework. By applying these ideas to a rather different subject matter, and in a way that figurational sociologists of sport have perhaps not previously done, this paper may illustrate a greater breadth to the approach than has previously been apparent.

\section{Acknowledgements}

I am grateful to Ken Sheard for his help with the design of the research project and data collection, and for his continued advice and support. I am also grateful to Ivan Waddington for his suggestion to consult the sociology of medicine literature on uncertainty, and to Martin Roderick for his thoughtful comments on an earlier draft of this work.

\section{References}

Adamson, C. (1997). Existential and clinical uncertainty in the medical encounter: An idiographic account of an illness trajectory defined by Inflammatory Bowel Disease and Avascular Necrosis. Sociology of Health and Illness, 19, 133-159.

Calnan, M. (1984). Clinical uncertainty: Is it a problem in the doctor-patient relationship? Sociology of Health and Illness, 6, 74-85.

Cockerham, W.C. (2004). Medical sociology ( $9^{\text {th }}$ Edition). Upper Saddle River, N.J.: Pearson/Prentice Hall. 
Conrad, P. (1987). The experience of illness: Recent and new directions. In J. Roth \& P. Conrad (Eds.), Research in the sociology of healthcare: A research annual, vol. 6. The Experience and management of chronic illness (pp. 1-31). Greenwich: Jai Press.

Curry, T.J. (1993). A little pain never hurt anyone: Athletic career socialization and the normalization of sports injury. Symbolic Interaction, 16, 273-290.

de Vaus, D.A. (1996). Surveys in social research (4th Edition). London: UCL Press.

Elias, N. (1978). What is sociology? London: Hutchison.

Fox, R.C. (1957). Training for uncertainty. In R.K. Merton, G. Reader \& P.L. Kendall (Eds.), The student-physician: Introductory studies in the sociology of medical education (pp. 207-241). Cambridge, MA: Harvard University Press.

Fox, R.C. (2000). Medical uncertainty revisited. In G.L. Albrecht, R. Fitzpatrick \& S.C. Scrimshaw (Eds.), The handbook of social studies in health and medicine (pp. 409-425). London: Sage.

Howe, P.D. (2004). Sport, professionalism and pain: Ethnographies of injury and risk. London: Routledge.

Hughes, R. \& Coakley, J. (1991). Positive deviance among athletes: The implications of overconformity to the sport ethic. Sociology of Sport Journal, 8, 307-325.

IRB (2008). Regulations relating to the game. International Rugby Board

Regulations. Retrieved March 11, 2008, from http://www.irb.com/mm/document/lawsregs/0/reg10a4feb08_4420.pdf

Light, D. (1979). Uncertainty and control in professional training. Journal of Health and Social Behavior, 20, 310-322.

Malcolm, D. (2006a). Unprofessional practice? The power and status of sport physicians. Sociology of Sport Journal, 23, 376-395. 
Malcolm, D. (2006b). Sports medicine: A very peculiar practice? Doctors and physiotherapists in elite English rugby union. In S. Loland, B. Skirstad \& I. Waddington (Eds.), Pain and injury in sport: Social and ethical analysis (pp. 165-181). London: Routledge.

Malcolm, D., \& Sheard, K. (2002). "Pain in the assets:" The effects of commercialization and professionalization on the management of injury in English rugby union. Sociology of Sport Journal, 19, 149-169.

Malcolm, D., Sheard, K. \& Smith, S. (2004). Protected research: Sports medicine and rugby injuries. Sport in Society, 2004, 7, 97-110.

Malcolm, D., Sheard, K. \& Smith, S. (2005). Protective equipment and the injury crisis in English rugby union. Football Studies, 8, 58-67.

May, T. (2001). Social research: Issues, methods and process (3rd Edition). Buckingham, UK: Open University Press.

McCrory, P. (1999). You can run by you can't hide: The role of concussion severity scales in sport. British Journal of Sports Medicine, 33, 297-280.

McCrory, P. (2001a). When to retire after concussion? British Journal of Sports Medicine, 35, 81-82.

McCrory, P. (2001b). Do mouthguards prevent concussion? British Journal of Sports Medicine, 35, 380-82.

McCrory, P., Johnston, K., Meeuwisse, W., Aubry, M., Cantu, R., Dvorak, J., GrafBaumann, T., Kelly, J., Lovell, M. and Schamasch, P. (2005). Summary and agreement statement of the $2^{\text {nd }}$ International Conference on Concussion in Sport, Prague 2004. British Journal of Sports Medicine, 39, 196-204.

Messner, M. (1992). Power at play: Sport and the problems of masculinity. Boston: MA Beacon Press. 
Murphy, P. \& Sheard, K. (2008) Boxing blind: Unplanned processes in the development of modern boxing. In D. Malcolm \& I. Waddington (Eds.), Matters of sport: Essays in honour of Eric Dunning (pp. 40-56). Abingdon: Routledge.

Nixon, H.L., II. (1992). A social network analysis of influences on athletes to play with pain and injuries. Journal of Sport and Social Issues, 16, 127-35.

Pike, E. \& Maguire, J. (2003) Injury in women’s sport: Classifying key elements of “risk encounters.” Sociology of Sport Journal, 20, 232-251.

Rafalovich, A. (2005). Exploring clinician uncertainty in the diagnosis and treatment of Attention Deficit Hyperactivity Disorder. Sociology of Health and Illness, 27, 305-323.

RFU (1998). The RFU handbook of safe rugby. London: A\&C Black.

Roderick, M. (2004). English professional soccer players and the uncertainties of injury. In K. Young (Ed.), Sporting bodies, damaged selves: sociological studies of sports related injuries (pp. 137-49). Oxford: Elsevier.

Roderick, M. (2006a). The sociology of pain and injury in sport: Main perspective and problems. In S. Loland, B. Skirstad, \& I. Waddington (Eds.), Pain and injury in sport: Social and ethical analysis. (pp. 17-33). London: Routledge.

Roderick, M. (2006b). The work of professional football: A labour of love? London: Routledge.

Roderick, M., Waddington, I. \& Parker, G. (2000). Playing hurt: Managing injuries in English professional football. International Review for the Sociology of Sport, 35, 165-180.

Safai, P. (2003). Healing the body in the "culture of risk:” Examining the negotiations of treatment between sport medicine clinicians and injured athletes in Canadian intercollegiate sport. Sociology of Sport Journal, 20, 127-46. 
Safai, P. (2004). Negotiating with risk: Exploring the role of the sports medicine clinician. In K. Young (Ed.), Sporting bodies, damaged selves: sociological studies of sports related injuries (pp. 269-288). Oxford: Elsevier.

Schwarz, A. (2007). Silence on Concussions Raises Risks of Injury. The New York Times, 15 September 2007.

Stroh, M. (2000). Qualitative interviewing. In D. Burton (Ed.), Research training for social scientists: A handbook for postgraduate researchers (pp.196-214). London: Sage.

Theberge, N. (2006). “It’s not about health, it’s about performance.” Sport medicine, health, and the culture of risk in Canadian sport. In J. Hargreaves and P. Vertinsky (Eds.), Physical culture, power, and the body (pp. 176-194). London: Routledge

Theberge, N. (2008). The integration of chiropractors into health care teams: A case study from sports medicine. Sociology of Health and Illness, 30, 19-34.

Waddington, I. (2000). Sport, health and drugs: A critical sociological perspective. London and New York: E \& FN Spon.

Waddington, I., \& Roderick, M. (2002). The management of medical confidentiality in English professional football clubs: Some ethical problems and issues. British Journal of Sports Medicine, 36, 118-123.

Walk, S.R. (1997). Peers in pain: The experiences of student athletic trainers. Sociology of Sport Journal, 14, 22-56.

Yair, G. (2007). Existential uncertainty and the will to conform: The expressive basis of Coleman's rational choice paradigm. Sociology, 41, 681-698. 
Young, K. (1993) Violence, risk and liability in male sports culture. Sociology of Sport Journal, 10, 373-96.

Young, K. \& White, P. (2000). Researching sports injury: Reconstructing dangerous masculinities. In J. McKay, M. Messner \& D. Sabo (Eds.), Masculinities, gender relations and sport (pp. 108-127). London: Sage.

Young, K. (2004) Sports-related pain and injury: Sociological notes. In K. Young (Ed.), Sporting bodies, damaged selves: sociological studies of sports related injuries (pp. 1-28). Oxford: Elsevier. 


\section{Notes}

1 Doctors may also keep patients in a state of “optimistic uncertainty” as a way of facilitating patient compliance.

2 Clubs in the top two Leagues largely have full-time coaching and playing staffs. Below this clubs may have one or two full-time players (often playercoaches or youth development officers), supported by paid, but part-time players.

3 Clause 10.1.3 states that the minimum period of absence is mandatory for age group rugby. 